\title{
Spinodal dynamics in cluster aggregation
}

\author{
M. CARPINETI and M. GIGLIO
}

Dipartimento di Fissica, Universitd di Milano, via Celoria 16, 20133 Milano, Italy

\begin{abstract}
We report small angle static light scattering measurements on colloidal aggregation at high monomer volume fractions. The structure factor $S(q)$ exhibits a pronounced maximum at a finite wave vector $q_{m}$ at any stage during the aggregation reaction. In the later stages the structure factor scales according to a universal law similar to that of spinodal decomposition dynamics: $S\left(q / q_{m}\right)=q_{m}^{-d} F\left(q / q_{m}\right)$. At variance with spinodal decomposition, where $d=3$, we find $d=d_{f}$, the fractal dimension of colloidal clusters:

The aggregation stops when the peak position reaches a certain value $q^{*}$ which depends on the monomer concentration. The terminal stage occurs when all the sample is filled with close packing fractal clusters of size $R \propto 1 / q^{*}$
\end{abstract}

\section{Introduction}

A binary solution, when quenched inside the coexistence curve undergoes phase separation. The kinetics of this non-equilibrium process has been actively investigated both theoretically [1-4] and experimentally [5-9]. In particular great effort has been produced to understand spinodal decomposition, the process of phase separation taking place in thermodynamically unstable solutions.

A completely different process where many works have been produced in the past years $[10]$ is that of colloidal aggregation. In this 
work we show that this phenomenon exhibits features very similar to that of spinodal decomposition.

The mechanism of spinodal decomposition can be explained by the fact that the thermodynamically unstable states inside the coexistence curve are characterized by a negative compressibility and this leads to a negative diffusion coefficient. Thus fluctuations inside the fluid grow instead of decaying. In particular it can be shown that at each instant there is a typical wavelength for which the fluctuations grow in the fastest possible way. Due to this spatial periodicity of the composition variation, a system which is phase separating following spinodal decomposition produces a ring shaped scattering pattern. The intensity and radius of the ring change in time as the system moves towards thermodynamic equilibrium. The dynamic of phase separation has been demonstrated to be common to very different physical systems, and a ring shaped scattering pattern, exhibiting features similar to that of spinodal decomposition, has been observed also in processes like the growth of cluster of glass [11] or the cristallization of colloids [12].

Referring now to the phenomenon of colloidal aggregation, it is well known that colloidal particles in water present a surface charge that prevent them from aggregating. The interaction potential depends both on coulombic repulsion and on Van der Waals attraction. By adding some electrolyte to the solution it is possible to screen the surface charge and to reduce the potential barrier untill only the diffusive motion of the particles limits the aggregation process.

We have studied with low angle static light scattering the process of aggregation in very dense colloidal solutions. As it is well known, the random kinetic growth produces complex structures characterized by fract.al morphology and, for dilute samples, the light scattered by a solution of fractal aggregates has a maximum for $q=0$ which is proportional to the weight average cluster mass. In spite of that we have observed for the first time a ring shaped scattering pattern whose radius, as in the case of spinodal decomposition, collapses to zero as time goes on. Furthermore, in the late stages of aggregation the intensity distribution closely follows the dynamics of spinodal decomposition [13]. We have found that the structure factor $S(q)$ scales according to the equation

$$
S\left(q / q_{m}, t\right)=q_{m}^{-d_{f}}(t) F\left(q / q_{m}\right)
$$

Here $q_{m}$ is the peak position, $F\left(q / q_{m}\right)$ is a time independent scaling function and $d_{f}$ is the fractal dimension. It should be pointed out 
that for spinodal decomposition the same relation holds but with $d=3$ instead of $d_{f}$.

\section{Experimental methods}

Aggregation has been induced by adding divalent salt $\left(\mathrm{MgCl}_{2}\right)$ to polystyrene spheres $(0.019 \mu$ in diameter $)$ in a water and deuterated water mixture used for matching the polystyrene density to the solvent and avoid differential sedimentation. Measurements have been performed at different monomer concentrations ranging from $8.25 \times 10^{12} \mathrm{~cm}^{-3}$ to $8.25 \times 10^{14} \mathrm{~cm}^{-3}$. The salt concentration has been chosen slightly below the DLCA value so to have sufficently slow reaction rates in spite of the high volume fractions used.

The low angle scattering setup, has been described in previous works $[14,15]$ and is sketched in Fig. 1.

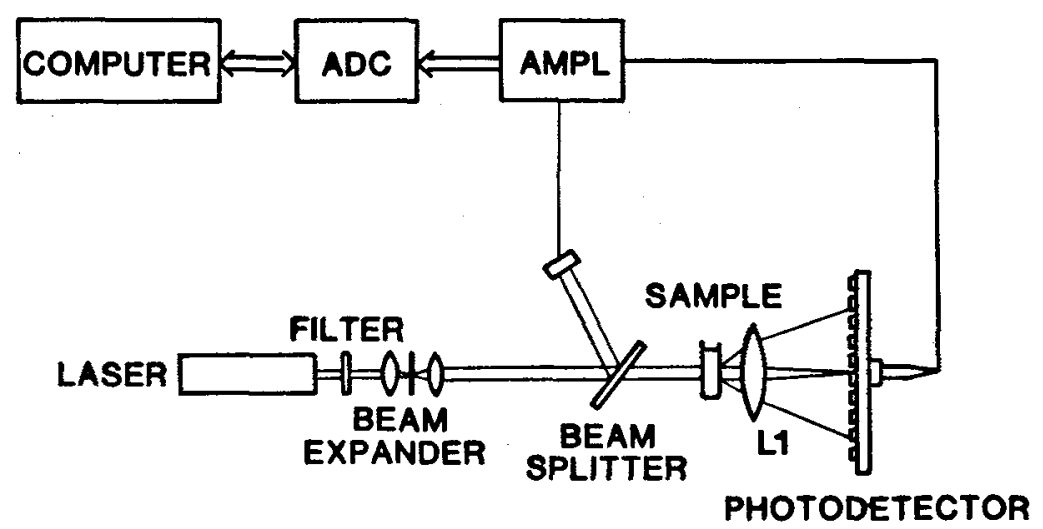

Fig. 1 - Experimental setup

A collimated beam impinges on a cuvette containing the aggregating sample. The light scattered by the sample together with the transmitted beam are collected by the lens $L 1$. In the back focal plane a multi element photodetector is placed. The elements are shaped as concentric quarters of an annulus and the focal plane arrangement realizes a corrispondence between the scattering angles and the radii 
of the sensing elements. The transmitted beam is focused into a pinhole in the center of the sensor behind which a photodiode is placed. The transmitted light is normalized to the power of the main beam, brought to an additional photodiode by a beam splitter before the cell.

Experimental results

In Fig. 2 we show a $\log -\log$ plot of intensity distributions taken at various times after the start of the aggregation for $c_{0}=3.3 \times$ $10^{14} \mathrm{~cm}^{-3}$.

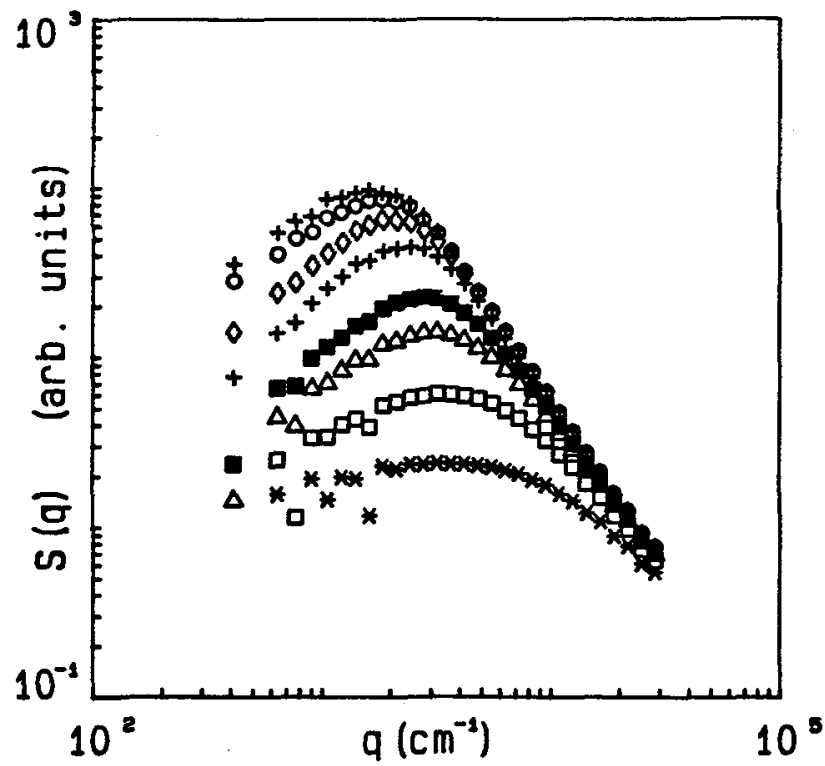

Fig. 2 - Log-log plot of intensity distributions taken at various times during the aggregation.

At variance with all the previous measurements on fractal aggregates, the intensity distributions do not have the maximum for $q \simeq 0$, but exhibit a pronounced peak for $q=q_{m}$. It is evident that the peak height increases in time and its position moves to smaller $q$ values. This behaviour is extremely similar to that observed for spinodal decompositon $[6,8,9]$.

Also it should be noticed that all the curves collapse onto the same asymptotic line at high $q$. The power law behaviour at high $q$ values 
is a typical feature of colloidal aggregates, the slope of the curve in a $\log -\log$ plot being the fractal dimension of the clusters $\left(d_{f}=\right.$ $1.9 \pm 0.1$ ). Furthermore the fact that only one asymptote is common to all the curves is a clear indication that no loss of mass in the scattering volume has occurred [14].

For all the reactions it is possible to distinguish three different stages. In the first stage the peak intensity grows very fast while the peak position moves slowly and the curves do not scale according to Eq. 1.

In the second (intermediate) phase the curves do follow this law as shown in Fig. 3.

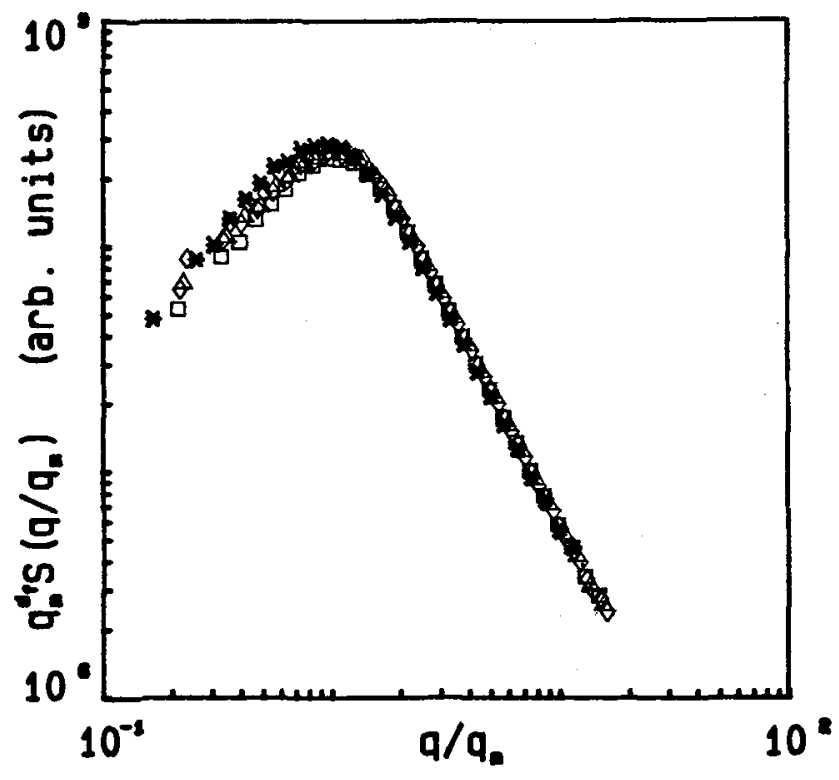

Fig. 3-Log-log plot of the scaled functions $S\left(q / q_{m}, t\right) q_{m}^{d_{f}}$

The third (terminal) stage of the aggregation is characterized by the fact that the reaction stops when the peak position has reached a certain value $q^{*}$ and we have evidence that the peak position at this stage is related to the monomer concentration used. The explanation of this phenomenon directly follows from the fractal nature of the aggregates. Infact the density of a fractal cluster decreases with its size, since the volume scales as $R_{g}^{3}$ while the mass scales as $R_{g}^{d_{f}}$. 
Therefore, it is easy to demonstrate that for every monomer concentration there should be a maximum value $R_{\max }$ for the average gyration radius at which all the fluid volume is filled with fractals. From simple calculations one can find that for $d_{f} \simeq 2$

$$
R_{\max }=\frac{1}{4 c_{0} R_{0}^{2}}
$$

In Fig. 4 we show the terminal curves for various monomer concentrations. Notice that the terminal peak position moves to higher $q$ values as the concentration is increased.

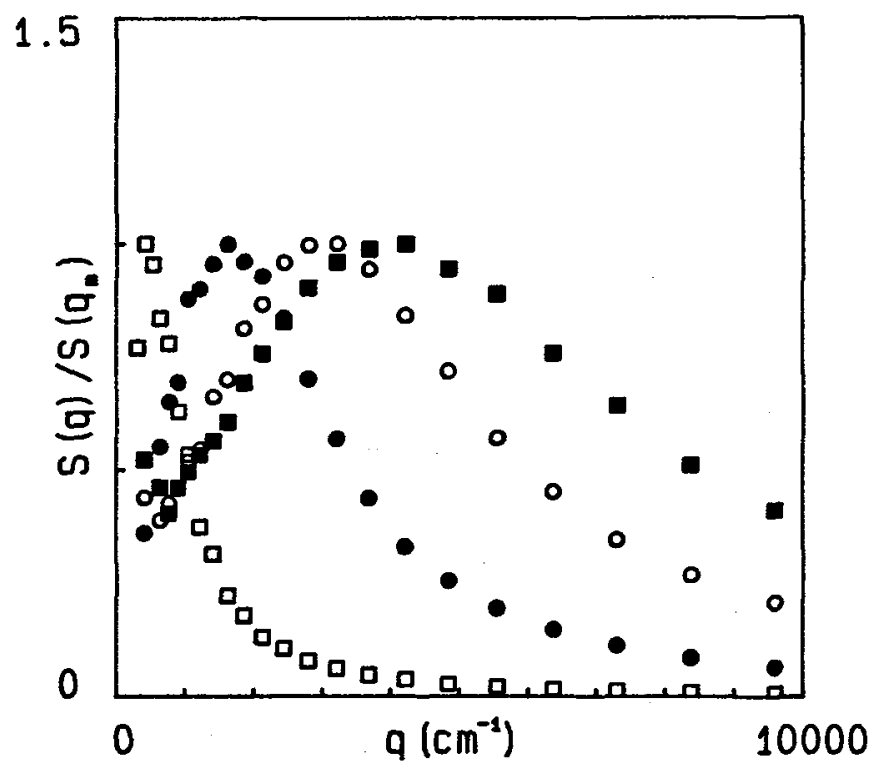

Fig. 4 - Terminal curves for different monomer concentrations ranging from $c_{0}=8.25 \times 10^{13} \mathrm{~cm}-3\left(\right.$ a) to $c_{0}=8.25 \times 10^{14} \mathrm{~cm}^{-3}(\mathbf{a})$.

For brevity we will not report here the plot of $R_{\max }$ versus $c_{0}$. Our data, however, are in very good agreement with Eq. 2 taking $R_{\max }=$ $\frac{1}{q^{*}}$.

Incidentally, for the lowest concentration used $\left(8.25 \times 10^{12} \mathrm{~cm}^{-3}\right)$ the scattered peak kept moving to smaller q-values untill it eventually shifted outside the experimental range. 


\section{Conclusions}

We have presented evidence of some similarities between spinodal decomposition and colloidal aggregation in dense solutions. These surprising analogies seem to indicate that some common mechanism should be operative in both phenomena. In particular, the presence of a peak in the structure factor is a clear indication that the clusters are arranged fairly regularly in space, thus creating a semi-random density modulation inside the fluid whose wavelength changes in time. The length scale of the spatial ordering at length scales is roughly $1 / q_{m}$. The growth of the clusters occurs at the expences of neighboring depletion regions, and this leads to the existence of a minimum distance between clusters growing with the cluster dimensions. Furthermore, the fractality of the clusters and in particular the fact that their density decreases with the distance from their core, produces a density modulation inside the solution, as in the case of spinodal decomposition.

The intermediate stage, when the curves can be scaled according to equation 1 occurs when the system can be described with a unique length, proportional to the average cluster radius.

Finally, the terminal stage occurs when the clusters have filled all the available space. The peak position does not change any more and is simply related to the mean distance between the aggregates, that at this stage is exactly the cluster diameter.

\section{Acknowledgments}

We thank V. Degiorgio and R. Piazza for helpful conversations. This work has been supported by the Italian Ministero dell'Università e della Ricerca Scientifica e Tecnologica (MURST) and the Consiglio Nazionale delle Ricerche (CNR).

\section{References}

[1] J.S. Langer in Flutuations, Instabilities, and Phase Transitions ed. by Tormod Riste (Plenum Press, New York and London) pp. 19-42.

[2] K. Binder, D. Stauffer, Adv. Phys. 25, 343 (1976).

[3] K. Binder, C. Billotet and P. Mirold, Z. Phys. B24, 407 (1976). 
[4] H. Furukawa, Adv. Phys, 34, 703 (1985).

[5] F. S. Bates and P. Wiltzius, J. Chem. Phys. 91, 3258 (1989).

[6] P. Wiltzius, F. S. Bates and W. R. Heffner, Phys. Rev. Lett. 60, 1538 (1988).

[7] J. S. Huang, W. I. Goldburg, and A. W. Bjierkaas, Phys. Rev. Lett. 32, 921 (19T4).

[8] Y. C. Chou and W. I. Goldburg, Phys. Rev. A. 23, 858 (1981).

[9] N. C. Wong and C. M. Knobler, Phys. Rev. A 24, 3205 (1981).

[10] See for example: The Fractal Approach to Heterogeneous Chemistry edited by D. Avnir (Wiley, New York, 1989).

[11] V. Degiorgio, G. P. Banfi, G. Righini, and A. Rennie, Appl. Phys. Lett. 57, 2879 (1990).

[12] K. Schatzel and B. J. Ackerson, Phys. Rev. Lett., 68, 337 (1992).

[13] M. Carpineti, and M. Giglio Phys. Rev. Lett. 68, 3327, (1992).

[14] M. Carpineti, F. Ferri, M. Giglio, U. Perini, and E. Paganini, Phys. Rev. A, 42, 7347 (1990).

[15] D. Asnaghi, M. Carpineti, M. Giglio, and M. Sozzi, Phys. Rev. A, 45, 1018 (1992). 